\title{
Improvement of overlapping hidradenitis suppurativa and ankylosing spondylitis after the introduction of adalimumab
}

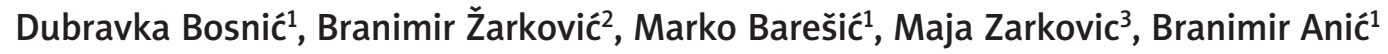 \\ ${ }^{1}$ Division of Clinical Immunology and Rheumatology, Department of Internal Medicine, University of Zagreb, School of Medicine, \\ University Hospital Center Zagreb, Zagreb, Croatia \\ 2Division of Rheumatology, Physical Medicine and Rehabilitation, General Hospital "Dr. Josip Benčević”, Slavonski Brod, Croatia \\ ${ }^{3}$ Division of Infectious Diseases, Dermatology and Venerology, General Hospital "Dr. Josip Benčević”, Slavonski Brod, Croatia
}

\begin{abstract}
Hidradenitis suppurativa is a chronic inflammatory disorder characterized by occlusion of the follicular pilosebaceous units of the skin. The treatment options are sometimes very limited and unpleasant odor and abundant drainage complicate the disease. Ankylosing spondylitis is a form of seronegative spondyloarthritis with predominantly axial but also peripheral joint involvement. Both of the conditions lower the patient's quality of life and affect everyday activities.

We describe a 39-year-old male patient with both diseases treated with different medications with only a modest result. After the initiation of a tumor necrosis factor $\alpha$ (TNF- $\alpha$ ) inhibitor (adalimumab) the patient experienced first the musculoskeletal and later on the skin improvement. The introduction of TNF- $\alpha$ inhibitors should be considered early in the treatment of overlapping hidradenitis suppurativa and the spondyloarthritis spectrum of conditions. Available medical data confirm the positive results and beneficial effect on disease course, activity and, most importantly, quality of life.
\end{abstract}

Key words: ankylosing spondylitis, adalimumab, hidradenitis suppurativa.

\section{Introduction}

Hidradenitis suppurativa (HS) also known as acne inversa, is a chronic, recurrent, inflammatory skin disease characterized by painful nodules, sinuses, abscesses, draining fistulas and scarring lesions occurring mainly in intertriginous areas of the axillas, groin, perianal, perineal and inframammary regions [1]. The prevalence of the disease is between $1-4 \%$ and it most commonly affects younger adults with a female predominance [2]. The risk factors associated with the onset and exacerbation of the disease are positive family history of the disease, obesity, smoking and mechanical friction [3]. The severity of HS can be classified with the Hurley classification [4] or Sartorius score $[5,6]$. The diagnosis is primarily clinical and skin biopsy is rarely required [7]. Hidradenitis suppurativa is associated with several diseases such as inflammatory bowel disease, endocrine disorders, metabolic syndrome and spondyloarthropathies [8-15]. Treatment depends on clinical stage and includes non-pharmacologic, pharmacologic and surgical procedures [16]. General measures are local hygiene, weight reduction, smoking cessation, and avoidance of skin trauma. Pharmacologic treatment includes topical and systemic antibiotics, intralesional corticosteroids, hormonal therapy and biologic therapy (tumor necrosis factor $\alpha$ [TNF- $\alpha]$ inhibitors) $[17,18]$.

Ankylosing spondylitis (AS) is a form of seronegative spondyloarthritis (SpA) and affects mostly younger male patients with predominantly axial but also peripheral joints and extra-articular involvement [19]. Ankylosing spondylitis is associated with HLA B27 antigen and the prevalence of the disease is between 0.2 and $1.2 \%$. The disease is manifested by inflammatory back pain and prolonged spinal stiffness. It is worsened by rest and usually improved by the use of nonsteroidal

\section{Address for correspondence}

Marko Barešić, Division of Clinical Immunology and Rheumatology, Department of Internal Medicine, University of Zagreb,

School of Medicine, University Hospital Center Zagreb, Zagreb, Croatia, e-mail: markobaresic@gmail.com

Submitted: 29.10.2016, Accepted: 13.12.2016 
Table I. Laboratory findings

\begin{tabular}{|c|c|c|}
\hline & $\begin{array}{c}\text { Before the } \\
\text { treatment } \\
\text { with } \\
\text { adalimumab }\end{array}$ & $\begin{array}{l}\text { Ten months } \\
\text { after the } \\
\text { introduction of } \\
\text { adalimumab }\end{array}$ \\
\hline ESR (ref. 4-24 mm/h) & 94 & 16 \\
\hline CRP (ref. < 5 mg/l) & 126.8 & 10.5 \\
\hline WBC (ref. 3.4-9.7 × 109/l) & 26.0 & 24.0 \\
\hline Hb (ref. 119-157 g/l) & 116 & 142 \\
\hline Trc (ref. 158-424 × 109/I) & 602 & 408 \\
\hline Routine biochemistry & normal & normal \\
\hline RF, ACPA & negative & - \\
\hline Complement levels & normal & - \\
\hline ANA, ENA & negative & - \\
\hline HLA typing & $\begin{array}{l}\text { B27 and DR4 } \\
\text { positive }\end{array}$ & - \\
\hline Blood and urine culture & sterile & - \\
\hline $\begin{array}{l}\text { BASDAI (>4 = active } \\
\text { disease) }\end{array}$ & 6.8 & 2.0 \\
\hline $\begin{array}{l}\text { BASFI } \\
(>4=\text { impaired function) }\end{array}$ & 5.7 & 2.2 \\
\hline DLQI & $\begin{array}{c}22 \\
\text { (extremely } \\
\text { large burden on } \\
\text { patient's life) }\end{array}$ & $\begin{array}{c}7 \\
\text { (moderate } \\
\text { burden on } \\
\text { patient's life) }\end{array}$ \\
\hline
\end{tabular}

ESR - erythrocyte sedimentation rate; CRP - C-reactive protein WBC - white blood cells; Hb - haemoglobin; Trc - thrombocytes; RF - rheumatoid factor; ACPA - anti-citrullinated peptide antibody; HLA - human leukocyte antigen; ANA - antinuclear antibodies, ENA - extractable nuclear antigens; BASDAI - Bath Ankylosing Spondylitis Disease Activity Index; BASFI - Bath Ankylosing Spondylitis Function Index; DLQI - dermatology life quality index anti-inflammatory drugs (NSAIDs) and with activity. The Assessment of SpondyloArthritis International Society (ASAS) developed new criteria for classification of both axial and peripheral SpA. Diagnosis of AS is based upon a combination of clinical, laboratory and imaging findings [20]. Management includes physiotherapy, analgesics, NSAIDs and biologic therapy. Physiotherapy is the most important non-pharmacological treatment in AS. NSAIDs in anti-inflammatory doses relieve pain and stiffness. Tumor necrosis factor $\alpha$ blockers are indicated in patients after failure of conventional treatment [20].

\section{Case presentation}

A 39-year-old overweight (body mass index BMI-40.3) man had a 20-year history of HS with skin lesions worsening over time. The HS was classified as stage III according to the Hurley classification [4]. The patient had previously been treated with topical and oral antibiotics with a partial clinical improvement, frequent recurrences and he often missed recommended dermatologic follow-ups.

The patient's medical history included progressive loss of vision for which he was admitted to the Ophthalmology unit and diagnosed with panuveitis of the right eye, intermediate uveitis of the left eye, retinal periphlebitis and periarteritis with secondary glaucoma. He had been treated with oral and topical glucocorticoids, cycloplegic agents (mydriatics) and oral methotrexate with clinical improvement. A brain MRI revealed demyelinating lesions, cerebrospinal fluid examination was normal and oligoclonal bands were negative. The patient did not have further neurological deficits and diagnosis of multiple sclerosis was excluded.

In the period of the past few years the patient reported low back pain and morning stiffness lasting
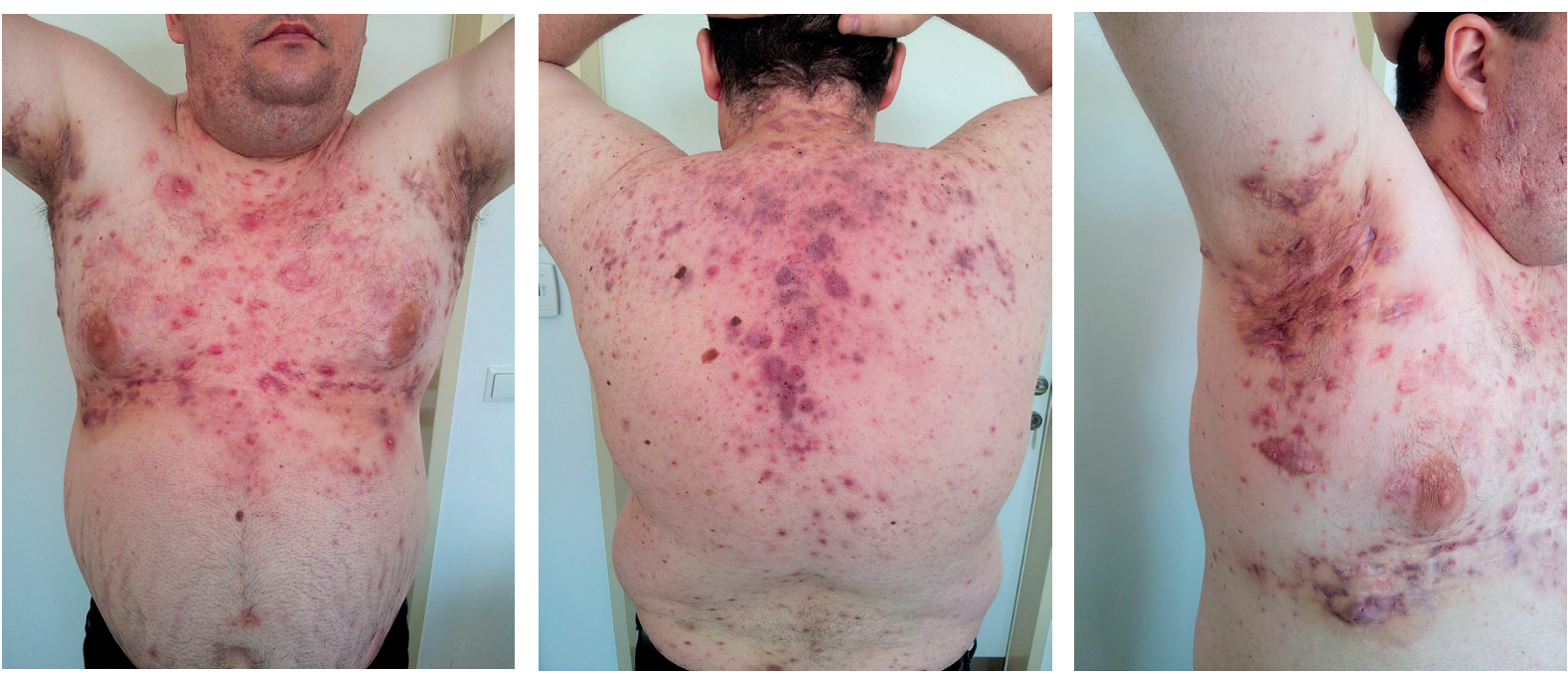

Fig. 1. Patient before the treatment with adalimumab. 
more than one hour. He was hospitalized due to prolonged low grade fever, fatigue, loss of body weight and worsening of HS. He presented with multiple painful inflamed draining nodules, fistulas and hypertrophic scars on the neck, trunk, axillary, inguinal, scrotal and sacralregions (Fig. 1). Blood examinations revealed elevated acute phase reactants with impaired full blood count (Table I). Blood and urine culture came back negative. The patient was seronegative (RF, ACPA, ANA/ENA) with normal levels components of complement (C3 and C4) and negative tests for coeliac disease with the presence of HLA-B27 and HLA-DR4 positivity. Further work-up excluded lymphoproliferative disorders and endoscopy proved no inflammatory bowel disease. Radiography of the spine and sacroiliac joints was conclusive for inflammatory changes and the patient was diagnosed with HLA-B27 positive AS without peripheral arthritis.

The patient was treated with a low dose of systemic glucocorticoids, full dose of NSAIDs and antibiotics with clinical improvement of inflammatory back pain and skin findings with normalization of acute phase reactants. At the first follow-up visit, the patient was feeling good with partial skin improvement and without pain, fever and neurological deficits. For the next three months he continued therapy with NSAIDs and reduced dosage of glucocorticoids, but despite this therapy he relapsed (primarily with symptoms of inflammatory back pain - BASDAI 6.8, BASFI 5.7 and elevated acute phase reactants). Due to high AS activity and an inadequate response to previous therapy, TNF- $\alpha$ inhibitor was introduced. The patient had no signs of latent tuberculosis and hepatitis serology came back negative so he received the first subcutaneous injection of adalimumab and continued $40 \mathrm{mg}$ every other week. Two months lat- er, the patient reported significant improvement of both AS and HS. He had minimal back pain, an better mobility without morning stiffness (BASDAI 2.0; BASFI 2.2). His $\mathrm{HS}$ has improved with a decrease in drainage from old lesions and a decrease in the formation of new lesions (Fig. 2). One year after the first adalimumab application the patient continues to have low disease activity of both diseases, low acute phase reactants (Table I) with no adverse reactions occurring during the treatment.

\section{Discussion}

The pathogenesis of HS is very complex and includes follicular occlusion, follicular rupture and an associated immune response. In the end the overall inflammatory process is seen as nodules, scarring tissue and draining sinuses and fistulas. The cytokines that drive inflammation in $\mathrm{HS}$ are TNF- $\alpha$, interleukin $1 \beta$ (IL-1 $\beta)$, IL-17 and IL-23 [21, 22].

The sequence of structural damage in spondyloarthritis consists of inflammation which leads to erosive bone destruction and replacement by repair tissue and finally to osteoproliferation with formation of syndesmophytes which is seen as irreparable damage of the patient's axial and sometimes peripheral skeleton. The process of inflammation is driven by proinflammatory cytokines including the most important TNF- $\alpha$ and also IL-17 and IL-23.

Tumor necrosis factor $\alpha$ is one of the connecting features of both diseases and serves as a cell signaling protein involved in systemic inflammation. Inhibition of this proinflammatory cytokine can lead to a benefit [23].

The patient we described suffered from HS and AS, both diseases equally disabling his everyday life activities and work. He underwent dermatologic and rheu-
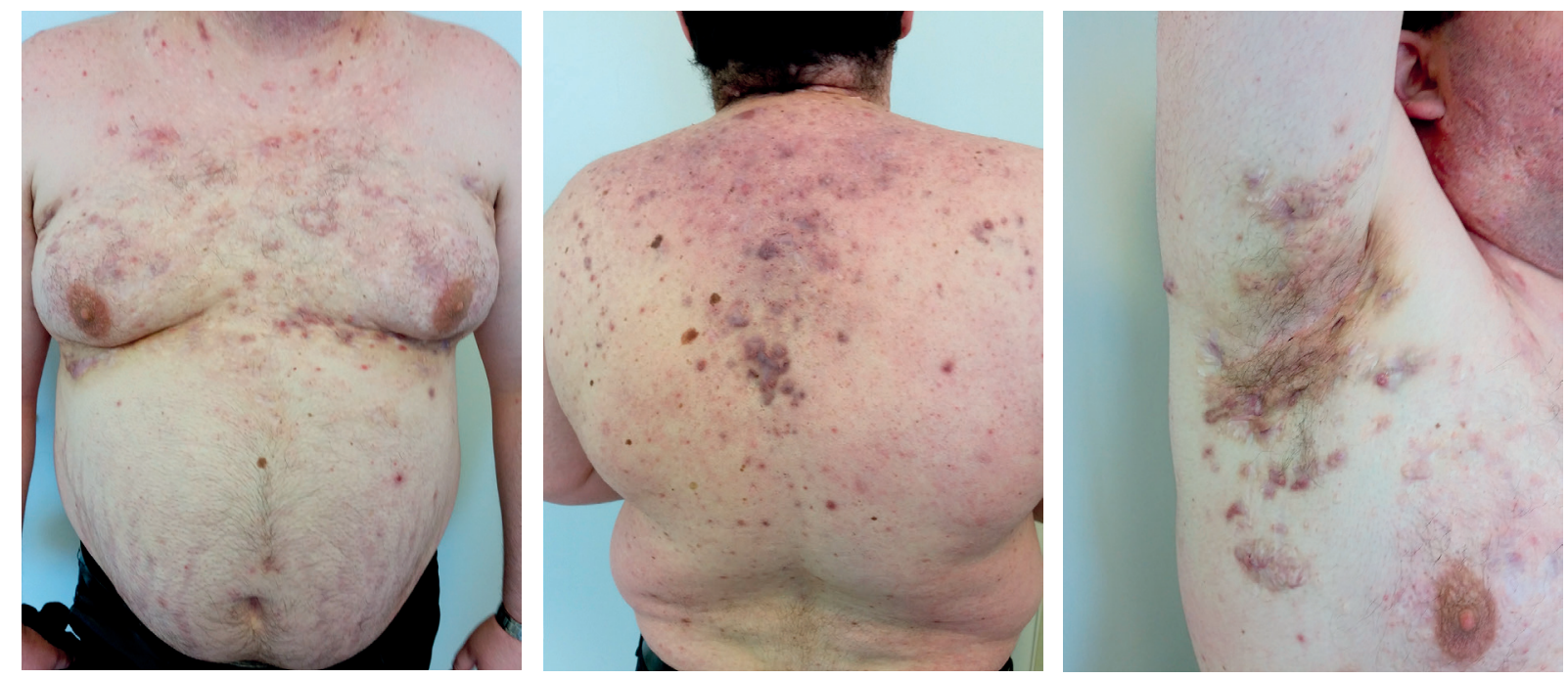

Fig. 2. Patient 10 months after the first adalimumab application. 
matologic check-ups and during the latter course of the diseases had more pronounced signs and symptoms of ankylosing spondylitis. Elevated acute phase reactants, inflammatory back pain and high disease activity indices were the reasons for the introduction of TNF- $\alpha$ inhibitor. After the careful screening for the active and latent infections the patient received first application of adalimumab according to the rheumatology regimen - $40 \mathrm{mg}$ every other week. The recommended dose of adalimumab for adult patients with moderate to severe HS is an initial dose of $160 \mathrm{mg}$ at week $0,80 \mathrm{mg}$ at week 2 , and $40 \mathrm{mg}$ every week starting at week 4 [17, 24].

The patient experienced a significant improvement of spondyloarthritis features but also HS skin lesions ameliorated despite the lower dosing regimen of adalimumab. The dermal changes did not completely disappear, but with the discontinuation of the inflammatory process the skin changes stopped producing unpleasant odor and secretion. The patient-reported outcome questionnaires and other measures improved significantly.

Adverse events in patients receiving adalimumab can be serious, but are not common taking into account its wide usage in rheumatological and ophthalmic indications. The most frequent infections include tuberculosis (primarily reactivation) and infections caused by viruses, fungi, or bacteria and also lymphoma, solid tissue cancers, cardiac failure and demyelinating central and peripheral nervous system disorders [25], so the patients should be monitored very carefully.

The current medical literature reports the effectiveness and desirability of treating HS with biological agents that inhibit TNF- $\alpha$ especially adalimumab and infliximab [26], but also others such as anakinra (anti-IL-1) [27] and ustekinumab (directed against IL-12 and IL-23) [28] may be a promising treatment strategy. Nevertheless further investigations are needed.

Spondyloarthropathies and hidradenitis suppurativa are two diseases that significantly affect patients' quality of life. BASDAI and BASFI are standard instruments for assessment of disease activity and efficacy of treatment of AS. The Dermatology Life Quality Index (DLQI) is an instrument used to measure the impact of skin disease on the quality of life of an affected person [30]. Treatment with TNF- $\alpha$ blockers improves both BASDAI, BASFI and DLQI [29, 30].

\section{Summary}

Timely introduction of targeted cytokine therapy can lead to the improvement of objective signs and symptoms of the hidradenitis suppurativa and ankylosing spondylitis as well as of the patient-reported outcome measures. Treatment strategy and dosing regimen should be tailored according to the more predominant features of the overlapping diseases. Although TNF- $\alpha$ inhibition brought overall improvement of both skin and musculoskeletal symptoms in the described patient several issues remain to be tackled. Optimal concomitant therapy in the overlapping disease (perhaps one of the DMARDs) and its effect on immunogenicity of the TNF- $\alpha$ inhibitor are unknown. The duration of TNF- $\alpha$ inhibition therapy when a patient reaches remission or low disease activity is one of the unsolved issues. Future casebased and similar studies of overlapping HS and AS should help in the approach to the mentioned problems.

The authors declare no conflict of interest.

\section{References}

1. Zouboulis CC, Desai N, Emtestam L, et al. European S1 guideline for the treatment of hidradenitis suppurativa/acne inversa. J Eur Acad Dermatol Venereol 2015; 29: 619-644.

2. Jemec GB, Heidenheim M, Nielsen NH. The prevalence of hidradenitis suppurativa and its potential precursor lesions. J Am Acad Dermatol 1996; 35: 191-194.

3. Micheletti RG. Hidradenitis suppurativa: current views on epidemiology, pathogenesis, and pathophysiology. Semin Cutan Med Surg 2014; 33 (3 Suppl): S48-50.

4. Revuz J. Hidradenitis suppurativa. J Eur Acad Dermatol Venereol 2009; 23: 985-998.

5. Sartorius K, Emtestam L, Jemec GB, et al. Objective scoring of hidradenitis suppurativa reflecting the role of tobacco smoking and obesity. Br J Dermatol 2009; 161: 831-839.

6. Barlev D, Eisen DB, Alikhan A. Hidradenitis suppurativa: a review with a focus on treatment data. Skin Therapy Lett 2015; 20: 1-8.

7. van der Zee HH, Jemec GB. New insights into the diagnosis of hidradenitis suppurativa: Clinical presentations and phenotypes. J Am Acad Dermatol 2015; 73 (5 Suppl 1): S23-S26.

8. Fimmel S, Zouboulis CC. Comorbidities of hidradenitis suppurativa (acne inversa). Dermatoendocrinol 2010; 2: 9-16.

9. Fauconier M, Reguiai Z, Colosio A, et al. AB0778 Vespa Study: Association Between Spondyloarthritis and Hidradenitis Suppurativa. Ann Rheum Dis 2015; 74: 1159.

10. Grassi W, Offidani AM, Blasetti P, et al. HLA-B27 negative ankylosing spondylitis and hidradenitis suppurativa: report of a case. Clin Rheumatol 1988; 7: 278-283.

11. Leybishkis B, Fasseas P, Ryan KF, et al. Hidradenitis suppurativa and acne conglobata associated with spondyloarthropathy. Am J Med Sci 2001; 321: 195-197.

12. Richette $\mathrm{P}$, Molto A, Viguier $\mathrm{M}$, et al. Hidradenitis suppurativa associated with spondyloarthritis - results from a multicenter national prospective study. J Rheumatol 2014; 41: 490-494.

13. Bruzzese V. Pyoderma gangrenosum, acne conglobata, suppurative hidradenitis, and axial spondyloarthritis: efficacy of anti-tumor necrosis factor $\alpha$ therapy. J Clin Rheumatol 2012; 18: $413-415$ 
14. Lim DT, James NM, Hassan S, et al. Spondyloarthritis associated with acne conglobata, hidradenitis suppurativa and dissecting cellulitis of the scalp: a review with illustrative cases. Curr Rheumatol Rep 2013; 15: 346.

15. Saraceno R, Babino G, Chiricozzi A, et al. PsAPASH: a new syndrome associated with hidradenitis suppurativa with response to tumor necrosis factor inhibition. J Am Acad Dermatol 2015; 72: e42-44.

16. Micheletti RG. An update on the diagnosis and treatment of hidradenitis suppurativa. Cutis 2015; 96 (6 Suppl): 7-12.

17. Martorell A, García FJ, Jiménez-Gallo D, et al. Update on Hidradenitis Suppurative (Part II): Treatment. Actas Dermosifiliogr 2015; 106: 716-724.

18. Gulliver W, Zouboulis CC, Prens E, et al. Evidence-based approach to the treatment of hidradenitis suppurativa/acne inversa, based on the European guidelines for hidradenitis suppurativa. Rev Endocr Metab Disord 2016; 17: 343-351.

19. Sieper J, Braun J, Rudwaleit M, et al. Ankylosing spondylitis: an overview. Ann Rheum Dis 2002; 61 Suppl 3: iii8-18.

20. Zochling J, van der Heijde D, Burgos-Vargas R, et al. ASAS/ EULAR recommendations for the management of ankylosing spondylitis. Ann Rheum Dis 2006; 65: 442-452.

21. Prens E, Deckers I. Pathophysiology of hidradenitis suppurativa: An update. J Am Acad Dermatol 2015; 73 (5 Suppl 1): S8-11.

22. Barlev D, Eisen DB, Alikhan A. Hidradenitis suppurativa: a review with a focus on treatment data. Skin Therapy Lett 2015; 20: $1-8$.
23. van der Zee HH, de Ruiter L, van den Broecke DG, et al. Elevated levels of tumour necrosis factor (TNF)- $\alpha$, interleukin (IL)-1 $\beta$ and IL-10 in hidradenitis suppurativa skin: a rationale for targeting TNF- $\alpha$ and IL-1 $\beta$. Br J Dermatol 2011; 164: 1292-1298.

24. Kimball AB, Kerdel F, Adams D, et al. Adalimumab for the treatment of moderate to severe Hidradenitis suppurativa: a parallel randomized trial. Ann Intern Med 2012; 157: 846-855.

25. Moul DK, Korman NJ. Severe Hidradenitis suppurativa treated with adalimumab. Arch Dermatol 2006; 142: 1110-1112.

26. Lee RA, Eisen DB. Treatment of hidradenitis suppurativa with biologic medications. J Am Acad Dermatol 2015; 73 (5 Suppl 1): S82-S88.

27. Tzanetakou V, Kanni T, Giatrakou S, et al. Safety and efficacy of anakinra in severe hidradenitis suppurativa: a randomized clinical trial. JAMA Dermatol 2016; 152: 52-59.

28. Blok J, Li K, Brodmerkel C, et al. Ustekinumab in hidradenitis suppurativa: clinical results and a search for potential biomarkers in serum. Br J Dermatol 2016; 174: 839-846.

29. Finlay AY, Khan GK. Dermatology Life Quality Index (DLQI) a simple practical measure for routine clinical use. Clin Exp Dermatol 1994; 19: 210-216.

30. Sotiriou E, Goussi C, Lallas A, et al. A prospective open-label clinical trial of efficacy of the every week administration of adalimumab in the treatment of hidradenitis suppurativa. J Drugs Dermatol 2012; 11 (5 Suppl): s15-20. 